Krist V. Gernaey, Jakob K. Huusom and Rafiqul Gani (Eds.), 12th International Symposium on Process Systems Engineering and 25th European Symposium on Computer Aided Process Engineering.

31 May - 4 June 2015, Copenhagen, Denmark. (C) 2015 Elsevier B.V. All rights reserved.

\title{
Control of Reaction Systems via Rate Estimation and Feedback Linearization
}

\author{
Diogo Rodrigues*, Julien Billeter and Dominique Bonvin \\ Laboratoire d'Automatique, EPFL, Lausanne, Switzerland \\ diogo.mateusrodrigues@epfl.ch
}

\begin{abstract}
The kinetic identification of chemical reaction systems often represents a time-consuming and complex task. This contribution presents an approach that uses rate estimation and feedback linearization to implement effective control without the use of a kinetic model. The reaction rates are estimated by numerical differentiation of reaction variants that are computed from measurements. The approach is illustrated in simulation through the temperature control of a continuous stirred-tank reactor.
\end{abstract}

Keywords: feedback linearization, rate estimation, numerical differentiation, reaction variants.

\section{Introduction}

Efficient control of reaction systems typically requires good kinetic models, whose identification however can be rather difficult and time consuming. As an alternative, one could try to infer the reaction rates directly from measurements, that is, without the help of a kinetic model, which can be done if the various rates can be decoupled (Mhamdi and Marquardt (2004)).

The concept of reaction variants and invariants has been proposed to decouple the dynamic effects in reaction systems, thereby facilitating their analysis and control (Asbjørnsen and Fjeld (1970); Asbjørnsen (1972)). A finer separation of the various dynamic effects in both homogeneous and heterogeneous open reaction systems has been proposed by Amrhein et al. (2010) and Bhatt et al. (2010), and reformulated recently as a linear transformation of the numbers of moles to so-called vessel extents by Rodrigues et al. (2015).

Although various control structures for continuous stirred-tank reactors based on reactions variants and extensive variables have been proposed throughout the years (Hammarström (1979); Georgakis (1986); Farschman et al. (1998); Dochain et al. (2009); Hoang et al. (2014)), there does not exist a systematic way of tackling the problem, in particular without the use of a kinetic model. The long-term objective of this research is the development of such a systematic control approach, which would utilize the linear transformation to vessel extents and control selected extents by adjusting the corresponding rates such as inlet flowrates or the power exchanged with the jacket.

This paper is a first step in that direction as it investigates the possibility of controlling chemical reactors without the explicit use of kinetic models. The reaction rates are estimated from concentration and temperature measurements via the concept of variants and then used via a feedbacklinearization scheme to control the reactor temperature by manipulating the amount of heat that is exchanged with the environment in a continuous stirred-tank reactor. 


\section{System description}

Let us consider an open homogeneous reactor with $S$ species, $R$ independent reactions, $p$ inlet streams and one outlet stream. The mole and heat balances can be written as follows (Rodrigues et al. (2015)):

$$
\underbrace{\left[\begin{array}{c}
\dot{\mathbf{n}}(t) \\
\dot{Q}(t)
\end{array}\right]}_{\dot{\mathbf{z}}(t)}=\underbrace{\left[\begin{array}{c}
\mathbf{N}^{\mathrm{T}} \\
(-\boldsymbol{\Delta H})^{\mathrm{T}}
\end{array}\right]}_{\mathscr{A}} \mathbf{r}_{v}(t)+\underbrace{\left[\begin{array}{c}
\mathbf{0}_{S} \\
1
\end{array}\right]}_{\mathbf{b}} q_{e x}(t)+\underbrace{\left[\begin{array}{c}
\mathbf{W}_{i n} \\
\check{\mathbf{T}}_{i n}^{\mathrm{T}}
\end{array}\right]}_{\mathscr{C}} \mathbf{u}_{i n}(t)-\omega(t) \underbrace{\left[\begin{array}{c}
\mathbf{n}(t) \\
Q(t)
\end{array}\right]}_{\mathbf{z}(t)}, \quad \underbrace{\left[\begin{array}{c}
\mathbf{n}(0) \\
Q(0)
\end{array}\right]}_{\mathbf{z}(0)}=\underbrace{\left[\begin{array}{c}
\mathbf{n}_{0} \\
Q_{0}
\end{array}\right]}_{\mathbf{z}_{0}},
$$

where $\mathbf{n}$ is the $S$-dimensional vector of numbers of moles, $Q(t)=m(t) c_{p}(t)\left(T(t)-T_{r e f}\right)$ the heat of the reaction mixture, $\mathbf{r}_{v}$ the $R$-dimensional vector of reaction rates, $q_{e x}$ the heat power that is exchanged with the jacket and the environment, $\mathbf{u}_{i n}$ the $p$-dimensional vector of inlet flowrates, $\omega(t):=\frac{u_{\text {out }}(t)}{m(t)}$ the inverse of the residence time, with $u_{\text {out }}$ the outlet flowrate and $m$ the mass in the reactor, $\mathbf{N}$ the $R \times S$ stoichiometric matrix, $\Delta \mathbf{H}$ the $R$-dimensional vector of heats of reaction, $\mathbf{W}_{\text {in }}$ the $S \times p$ inlet-composition matrix, $\check{\mathbf{T}}_{i n}$ the $p$-dimensional vector of inlet specific enthalpies, $V$ the reactor volume, $c_{p}$ the specific heat capacity, $T$ the temperature and $T_{r e f}$ a reference temperature. The state vector $\mathbf{z}$ and the vector $\mathbf{b}$ are both of dimension $S+1$, while the matrix $\mathscr{A}$ has dimension $(S+1) \times R$ and the matrix $\mathscr{C}$ has dimension $(S+1) \times p$.

\subsection{Transformation to reaction-variant states}

If $\operatorname{rank}(\mathscr{A})=R$, there exists a transformation matrix $\mathscr{T}$ of dimension $R \times(S+1)$ such that

$$
\mathscr{T} \mathscr{A}=\mathbf{I}_{R} .
$$

Applying the transformation $\mathscr{T}$ to Eq.(1) and defining $\mathbf{x}_{\mathbf{r}_{v}}(t):=\mathscr{T} \mathbf{z}(t)$ leads to

$$
\dot{\mathbf{x}}_{\mathbf{r}_{v}}(t)=\mathbf{r}_{v}(t)+(\mathscr{T} \mathbf{b}) q_{e x}(t)+(\mathscr{T} \mathscr{C}) \mathbf{u}_{i n}(t)-\omega(t) \mathbf{x}_{\mathbf{r}_{v}}(t), \quad \mathbf{x}_{\mathbf{r}_{v}}(0)=\mathscr{T} \mathbf{z}_{0} .
$$

The transformed states $\mathbf{x}_{\mathbf{r}_{v}}$ are reaction variants, with each state $x_{r_{v, i}}(i=1, \ldots, R)$ depending on the rate $r_{v, i}$, the manipulated variable $q_{e x}$, a combination of the inlet flowrates $\mathbf{u}_{i n}$, and $\omega$ the inverse of the residence time. To be applicable, the transformation $\mathscr{T}$ requires that at least $R$ elements of the vector $\mathbf{z}$ be measured (Rodrigues et al. (2015)). Note that transformations based on $[\mathscr{A} \mathbf{b}]$ or $\left[\begin{array}{lll}\mathscr{A} & \mathbf{b} & \mathbf{z}_{0}\end{array}\right]$ could also be used, but they would require stricter rank conditions and thus more measured quantities, that is, $R+1$ or $R+2$ instead of $R$.

The proposed control scheme includes two steps, namely, the estimation of the reaction rates from the reaction-variant states $\mathbf{x}_{\mathbf{r}_{v}}$ and temperature control via feedback linearization.

\section{Control Problem}

The objective is to implement temperature control, that is, to control the heat signal $Q(t)$ to either the constant setpoint $Q_{s}$ or the reference trajectory $Q_{s}(t)$ by manipulating the exchanged heat power $q_{e x}(t)$. The reaction rates $\mathbf{r}_{v}(t)$ will be estimated without the use of a kinetic model using the measured quantities $\mathbf{z}(t), \mathbf{u}_{i n}(t), \omega(t)$ and the manipulated variable $q_{e x}(t)$.

\subsection{Estimation of reaction rates}

The estimation of $\mathbf{r}_{v}(t)$ proceeds via the differentiation of the reaction variants $\mathbf{x}_{\mathbf{r}_{v}}(t)$ that are obtained by transformation of $\mathbf{z}(t)$, or of subset of it (of dimension $S_{a}+1 \geq R$ ), and the knowledge of the quantities $q_{e x}(t), \mathbf{u}_{i n}(t)$ and $\omega(t)$.

Reformulating Eq.(3) yields the reaction rates

$$
\mathbf{r}_{v}(t)=\dot{\mathbf{x}}_{\mathbf{r}_{v}}(t)-(\mathscr{T} \mathbf{b}) q_{e x}(t)-(\mathscr{T} \mathscr{C}) \mathbf{u}_{i n}(t)+\omega(t) \mathbf{x}_{\mathbf{r}_{v}}(t) .
$$


Different transformations $\mathscr{T}$ that satisfy the condition in Eq.(2) can be found. An example is the Moore-Penrose pseudo-inverse of the matrix $\mathscr{A}$. However, when only noisy measurements of the state vector $\mathbf{z}$ are available, a better alternative is to consider an estimator in the maximumlikelihood sense, for which the transformation (2) is computed as

$$
\mathscr{T}=\left(\mathscr{A}^{\mathrm{T}} \boldsymbol{\Sigma}^{-1} \mathscr{A}\right)^{-1} \mathscr{A}^{\mathrm{T}} \boldsymbol{\Sigma}^{-1},
$$

where $\boldsymbol{\Sigma}$ is the $(S+1)$-dimensional variance-covariance matrix of the measurements. Note that the weighted transformation in Eq.(5) satisfies Eq.(2). The estimates $\hat{\mathbf{r}}_{v}(t)$ of the reaction rates given by Eq.(4) can be computed as described in Appendix A.

\subsection{Temperature control via feedback linearization}

The controller forces the heat signal $Q(t)$ to converge towards its reference trajectory $Q_{s}(t)$ at a desired rate. Defining the new input $v(t)$ to represent the right-hand side of the heat balance in Eq.(1) results in an integral relationship between the input $v(t)$ and the controlled variable $Q(t)$,

$$
\dot{Q}(t)=(-\Delta \mathbf{H})^{\mathrm{T}} \mathbf{r}_{v}(t)+q_{e x}(t)+\check{\mathbf{T}}_{i n}^{\mathrm{T}} \mathbf{u}_{i n}(t)-\omega(t) Q(t) \stackrel{!}{=} v(t) .
$$

Such an approach builds on feedback linearization, as shown in Figure 1. Solving Eq.(6) for $q_{e x}$ and replacing $\mathbf{r}_{v}$ by its estimate $\hat{\mathbf{r}}_{v}$ according to Eq.(12) in Appendix A gives the following expression for the manipulated variable:

$$
q_{e x}(t)=v(t)-(-\Delta \mathbf{H})^{\mathrm{T}} \hat{\mathbf{r}}_{v}(t)-\check{\mathbf{T}}_{i n}^{\mathrm{T}} \mathbf{u}_{i n}(t)+\omega(t) Q(t) .
$$

One can design a feedback controller that forces the control error $e(t):=Q_{s}(t)-Q(t)$ to converge exponentially to zero at the rate $\gamma$,

$$
\dot{e}(t)=-\gamma e(t), \quad e(0)=Q_{s}(0)-Q(0),
$$

by using the control law

$$
v(t)=\dot{Q}_{s}(t)+\gamma\left(Q_{s}(t)-Q(t)\right) .
$$

Note that this control law uses $\dot{Q}_{s}(t)$, which ideally requires prior knowledge of the reference signal $Q_{s}(t)$.

\section{Simulated example}

Consider the simulated example of the acetoacetylation of pyrrole in a homogeneous CSTR of constant volume with $S=4$ species (A: pyrrole; B: diketene; C: 2-acetoacetylpyrrole; D: dehydroacetic acid), $R=2$ reactions ( $\mathrm{A}+\mathrm{B} \rightarrow \mathrm{C}, 2 \mathrm{~B} \rightarrow \mathrm{D}$ ), $p=2$ inlets (of $\mathrm{A}$ and $\mathrm{B}$ ) and 1 outlet, the flowrate of which is adjusted to keep the volume constant (Ruppen et al. (1998)).

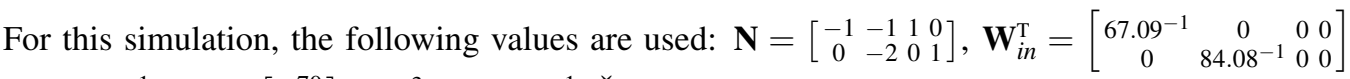
$\mathrm{kmol} \mathrm{kg}{ }^{-1}, \Delta \mathbf{H}=\left[\begin{array}{c}-70 \\ -50\end{array}\right] \times 10^{3} \mathrm{~kJ} \mathrm{kmol}^{-1}, \check{\mathbf{T}}_{i n}=\mathbf{0}_{p}$ at $T_{r e f}=298.15 \mathrm{~K}, r_{v, 1}=V k_{1} c_{A} c_{B}$ and $r_{v, 2}=$ $V k_{2} c_{B}^{2}$, where $\mathbf{c}(t)=\mathbf{n}(t) / V(t), k_{1}=A_{1} \exp \left(-\frac{E_{a, 1}}{R T}\right)$ and $k_{2}=A_{2} \exp \left(-\frac{E_{a, 2}}{R T}\right)$. The values of $A_{1}$, $A_{2}, E_{a, 1}, E_{a, 2}$, densities and specific heat capacities are adapted from Maria and Dan (2011). The volume is constant at $V=90.16 \mathrm{~L}$. Furthermore, it is assumed that the density and the specific heat capacity are constant, which results in the constant heat capacity $m c_{p}=129.5 \mathrm{~kJ} \mathrm{~K}^{-1}$.

The system is initially at steady state corresponding to the inputs $\bar{q}_{e x}=-4.9 \times 10^{3} \mathrm{~kJ} \mathrm{~min}^{-1}$ and $\overline{\mathbf{u}}_{i n}=\left[\begin{array}{l}\bar{u}_{i n, A} \\ \bar{u}_{i n, B}\end{array}\right]=\left[\begin{array}{l}40 \\ 15\end{array}\right] \mathrm{kg} \mathrm{min}^{-1}$, which gives the initial values $\mathbf{n}_{0}^{\mathrm{T}}=\left[\begin{array}{llll}0.833 & 0.093 & 0.143 & 0.028\end{array}\right]$ 


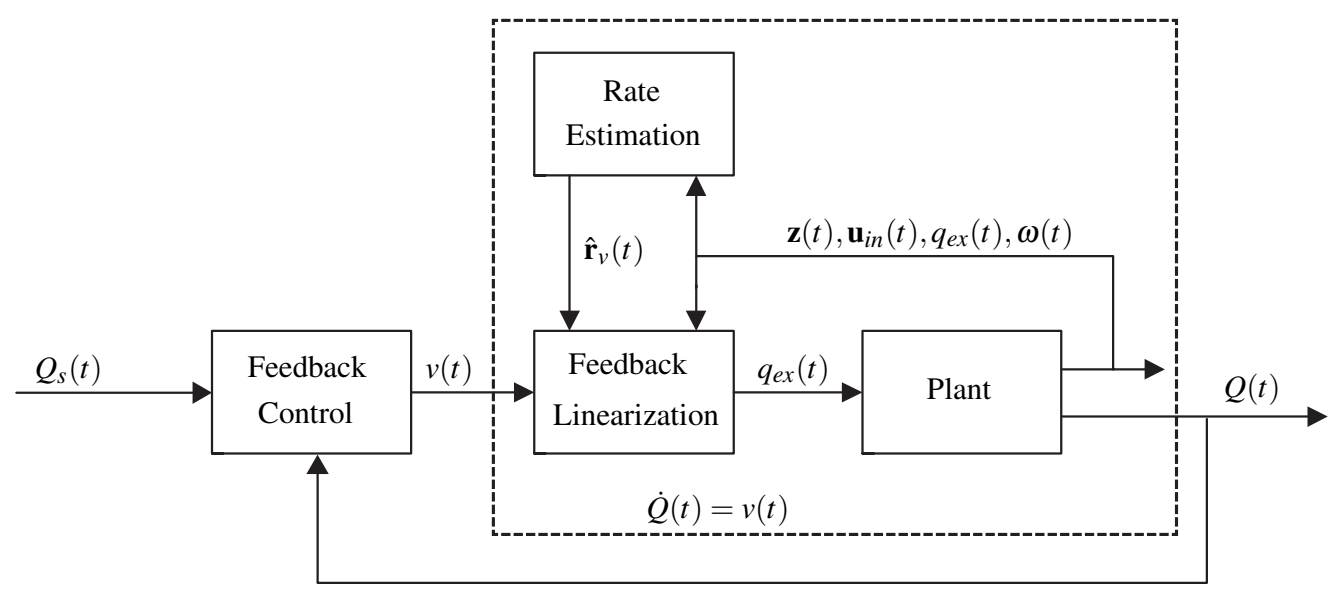

Figure 1: Temperature control based on feedback linearization and estimation of the reaction rates.
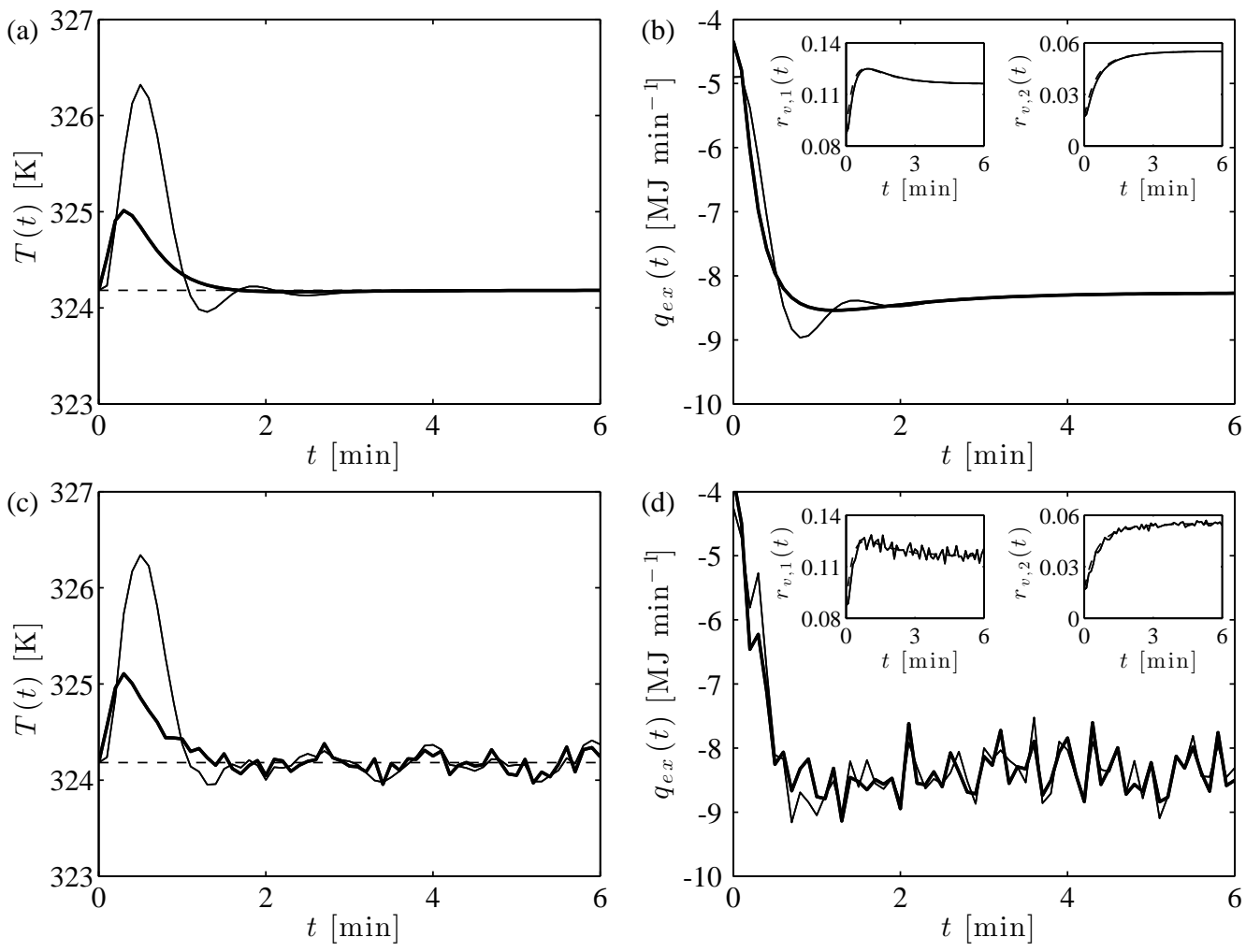

Figure 2: (a) and (c): Temperature profiles for feedback-linearization control (thick line) and PI control (thin line), with the setpoint shown by the dashed line; (b) and (d): Exchanged heat power

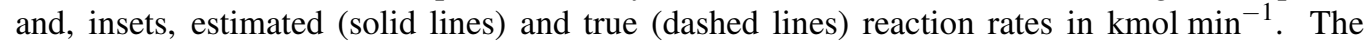
subfigures (a) and (b) show results without measurement noise, whereas (c) and (d) show results with measurement noise. 
$\mathrm{kmol}$ and $Q_{0}=3.37 \times 10^{3} \mathrm{~kJ}$ (corresponding to $T_{0}=324.2 \mathrm{~K}$ ). The reaction rates $\mathbf{r}_{v}$ are estimated from Eq.(12) in Appendix A. The control objective is to reject a $15 \mathrm{~kg} \mathrm{~min}^{-1}$ step disturbance in $u_{i n, B}$ by manipulating $q_{e x}(t)$.

Measurements of $\mathbf{z}, q_{e x}, \mathbf{u}_{i n}$ and $\omega$ are available at the sampling time $h_{s}=0.4 \mathrm{~s}$. It is assumed that the measurement errors in $q_{e x}, \mathbf{u}_{i n}$ and $\omega$ are negligible in comparison to those in $\mathbf{z}$, for which the standard deviation of the concentration measurements is $0.5 \%$ of the maximum concentration of each species and the standard deviation of the temperature measurements is $0.5 \mathrm{~K}$. This results in the variance-covariance matrix $\boldsymbol{\Sigma}=\operatorname{diag}\left(\left[\begin{array}{lll}0.004^{2} & 0.001^{2} 0.001^{2} 0.00025^{2} 65^{2}\end{array}\right]\right)$. A differentiation filter of order 1 and window size $q=25$ is used (Savitzky and Golay (1964)).

Feedback-linearization control using the exponential convergence rate $\gamma=5 \mathrm{~min}^{-1}$ is compared to PI control with the gain $K_{p}=5 \mathrm{~min}^{-1}$ and the integral time constant $\tau_{I}=0.2 \mathrm{~min}$. Figure 2 shows that the feedback-linearization scheme is able to reject the disturbance more quickly than the PI controller. However, if the standard deviation of the concentration measurements is larger than about $1 \%$ of the maximum concentration of each species, the estimated reaction rates become too imprecise or delayed (due to the choice of a larger window size $q$ ), and the advantage of feedback linearization over PI control is less clear (results not shown).

\section{Conclusions}

This paper has considered the control of the heat signal $Q$ (or temperature $T$ ) by manipulating the exchanged heat power $q_{e x}$ in an open homogeneous reactor. Control is implemented without the knowledge of a kinetic model. Instead, the reaction rates are (i) estimated via differentiation of reaction variants that are computed from measured states, and (ii) used in a feedback-linearization scheme that simplifies control design significantly. The parameters of the feedback-linearization controller are determined by readily available information, namely, the stoichiometry, the heats of reaction, the inlet composition and specific heat, and the inlet and outlet flow rates. Instead of linearizing the system around a given steady state, this controller implements feedback linearization that allows tracking a trajectory by forcing the control error to decay exponentially to zero. The resulting controller shows good performance for the case of frequent and precise concentration measurements of several species.

The controller requires $\operatorname{rank}(\mathscr{A})=R$, that is, at least as many measured quantities as there are reaction rates $\left(S_{a}+1 \geq R\right)$. The controller has two tunable parameters, namely, the exponential convergence rate $\gamma$ and the parameter of the differentiation filter (the number of samples $q$ in the case of the Savitzky-Golay filter) used for the rate estimation. These parameters need to be chosen to guarantee closed-loop stability. This study has shown that, at least in the case of low measurement noise, feedback linearization coupled to rate estimation can outperform PI control for the purpose of disturbance rejection.

\section{A. Appendix}

Let us approximate the derivative $\dot{\mathbf{x}}_{\mathbf{r}_{v}}(t)$ using a differentiation filter, such as the first-order filter proposed by Savitzky and Golay (1964), denoted as $\mathscr{D}_{q}\left(\mathbf{x}_{\mathbf{r}_{v}}, t\right)$, where $q$ is the window size expressed in number of samples in the time interval $[t-\Delta t, t]$, with $\Delta t:=(q-1) h_{s}$ and $h_{s}$ the sampling time. It can be shown that, since $\mathbf{x}_{\mathbf{r}_{v}}$ is Lipschitz continuous, $\mathscr{D}_{q}\left(\mathbf{x}_{\mathbf{r}_{v}}, t\right)$ can be reformulated as

$$
\mathscr{D}_{q}\left(\mathbf{x}_{\mathbf{r}_{v}}, t\right)=\sum_{k=0}^{q-2} b_{k+1} \int_{k}^{k+1} \dot{\mathbf{x}}_{\mathbf{r}_{v}}\left(t_{\xi}\right) d \xi
$$


with the weighting coefficients $b_{k+1}=\frac{6(q-1-k)(k+1)}{q\left(q^{2}-1\right)}>0$, such that $\sum_{k=0}^{q-2} b_{k+1}=1$, and $t_{\xi}:=t-$ $\Delta t+\xi h_{s}$. Replacing $\dot{\mathbf{x}}_{\mathbf{r}_{v}}$ by its expression in Eq.(3) gives

$$
\begin{aligned}
\mathscr{D}_{q}\left(\mathbf{x}_{\mathbf{r}_{v}}, t\right) & =\sum_{k=0}^{q-2} b_{k+1} \int_{k}^{k+1}\left(\mathbf{r}_{v}\left(t_{\xi}\right)+(\mathscr{T} \mathbf{b}) q_{e x}\left(t_{\xi}\right)+(\mathscr{T} \mathscr{C}) \mathbf{u}_{i n}\left(t_{\xi}\right)-\omega\left(t_{\xi}\right) \mathbf{x}_{\mathbf{r}_{v}}\left(t_{\xi}\right)\right) d \xi \\
& \approx \mathbf{r}_{v}(t)+\sum_{k=0}^{q-2} b_{k+1}\left((\mathscr{T} \mathbf{b}) q_{e x}\left(t_{k}\right)+(\mathscr{T} \mathscr{C}) \mathbf{u}_{i n}\left(t_{k}\right)-\omega\left(t_{k}\right) \mathbf{x}_{\mathbf{r}_{v}}\left(t_{k}\right)\right)
\end{aligned}
$$

where $t_{k}:=t-\Delta t+k h_{s}$.

The approximation in Eq.(11) is valid under the assumptions that $\mathbf{r}_{v}(t)$ is approximately constant in the time interval $[t-\Delta t, t]$ and the quantities $q_{e x}(t), \mathbf{u}_{i n}(t)$ and $\omega(t) \mathbf{x}_{\mathbf{r}_{v}}(t)$ are approximately constant in each time interval $\left[t_{k}, t_{k+1}[\right.$.

Defining the operator $\mathscr{W}_{q}(f, t):=\sum_{k=0}^{q-2} b_{k+1} f\left(t_{k}\right)$ for any function $f(t)$, rearranging Eq.(11) for $\mathbf{r}_{v}(t)$ and using measured quantities, denoted as $(\tilde{)})$, yields

$$
\hat{\mathbf{r}}_{v}(t)=\mathscr{D}_{q}\left(\tilde{\mathbf{x}}_{\mathbf{r}_{v}}, t\right)-(\mathscr{T} \mathbf{b}) \mathscr{W}_{q}\left(\tilde{q}_{e x}, t\right)-(\mathscr{T} \mathscr{C}) \mathscr{W}_{q}\left(\tilde{\mathbf{u}}_{i n}, t\right)+\mathscr{W}_{q}\left(\tilde{\omega} \tilde{\mathbf{x}}_{\mathbf{r}_{v}}, t\right)
$$

Eq.(12) approximates Eq.(4) for the case of measured quantities and can be used in Eq.(7).

\section{References}

M. Amrhein, N. Bhatt, B. Srinivasan, D. Bonvin, 2010, Extents of reaction and flow for homogeneous reaction systems with inlet and outlet streams, AIChE J., 56, 11, 2873-2886.

O. A. Asbjørnsen, 1972, Reaction invariants in the control of continuous chemical reactors, Chem. Eng. Sci., 27, 4, 709-717.

O. A. Asbjørnsen, M. Fjeld, 1970, Response modes of continuous stirred tank reactors, Chem. Eng. Sci., 25, 11, $1627-1636$.

N. Bhatt, M. Amrhein, D. Bonvin, 2010, Extents of reaction, mass transfer and flow for gas-liquid reaction systems, Ind. Eng. Chem. Res., 49, 17, 7704-7717.

D. Dochain, F. Couenne, C. Jallut, 2009, Enthalpy based modelling and design of asymptotic observers for chemical reactors, Int. J. Control, 82, 8, 1389-1403.

C. A. Farschman, K. P. Viswanath, B. E. Ydstie, 1998, Process systems and inventory control, AIChE J., 44, 8, 18411857.

C. Georgakis, 1986, On the use of extensive variables in process dynamics and control, Chem. Eng. Sci., 41, 6, 1471-1484.

L. G. Hammarström, 1979, Control of chemical reactors in the subspace of reaction and control variants, Chem. Eng. Sci., 34, 6, 891-899.

N. H. Hoang, D. Dochain, B. E. Ydstie, 2014, Partial inventory control of the CSTR via reaction-dependent generalized inventories, 19th IFAC World Congress, Cape Town, South Africa, 9123-9128.

G. Maria, A. Dan, 2011, Derivation of optimal operating policies under safety and technological constraints for the acetoacetylation of pyrrole in a semi-batch catalytic reactor, Comp. Chem. Eng., 35, 1, 177-189.

A. Mhamdi, W. Marquardt, 2004, Estimation of reaction rates by nonlinear system inversion, ADCHEM 2003, Hong Kong, China, 171-176.

D. Rodrigues, S. Srinivasan, J. Billeter, D. Bonvin, 2015, Variant and invariant states for reaction systems, Comp. Chem. Eng., 73, 1, 23-33.

D. Ruppen, D. Bonvin, D. W. T. Rippin, 1998, Implementation of adaptive optimal operation for a semi-batch reaction system, Comp. Chem. Eng., 22, 1, 185-189.

A. Savitzky, M. Golay, 1964, Smoothing and differentiation of data by simplified least squares procedures, Anal. Chem., 36, 8, 1627-1639. 